\title{
CONTROLE ESTATÍSTICO DE PROCESSO NA PRODUÇÃO DE REQUEIJÃO: ESTUDO DE CASO EM UM LATICÍNIO NO SUL DE MINAS GERAIS
}

\author{
Statistical process control in the production of Requeijão: a case \\ study in a dairy industry in the south of Minas Gerais state, Brazil
}

\author{
Gabriel Baldasso ${ }^{1 *}$, Raphael César de Souza Pimenta ${ }^{1}$, Eric Batista Ferreira ${ }^{1}$
}

\begin{abstract}
RESUMO
Por ser tipicamente brasileiro e muito útil no reprocessamento da muçarela, o requeijão se tornou um produto de alto consumo e produção no Brasil. Com o passar do tempo, o aumento da competitividade entre as indústrias de laticínios fez com que a produtividade do requeijão aumentasse. Contudo, é fundamental aumentar a produção do requeijão sem perder a qualidade. Sabe-se que uma forma de promover alta produtividade e alta qualidade em um processo é utilizando o Controle Estatístico da Qualidade (CEQ), que visa auxiliar o processo de produção a corrigir os erros identificáveis ao longo do processo, com intuito de elevar a rentabilidade da produção. Este trabalho apresenta a implantação do Controle Estatístico de Processo (CEP) em uma linha de produção de requeijão de uma indústria de laticínios no sul de Minas Gerais. Foram utilizadas cartas de controle para variáveis e índices de capabilidade, com o intuito de monitorar variáveis críticas do processo de produção do requeijão, com a finalidade de atender às especificações do Regulamento Técnico de Identidade e Qualidade (RTIQ). Após análise dos resultados, foi verificado que as variáveis do processo estão sob controle estatístico e são capazes de atender a norma especificada. Contudo, analisando a variabilidade dos dados, foram identificados padrões de não aleatoriedade, o que indica que a variabilidade do processo não se encontra sob controle estatístico. A principal causa apontada foi a baixa precisão dos dados coletados no processo produtivo, o que mostra a efetividade das ferramentas do CEP.

Palavras-chave: gestão da qualidade; derivados do leite; índice de capabilidade.
\end{abstract}

1 Universidade Federal de Alfenas, Rua Gabriel Monteiro da Silva, 700, Centro, 37130-001,Alfenas, MG, Brasil. E-mail:gabrielbaldassso@gmail.com

* Autor para correspondência

Recebido / Received: 26/01/2020

Aprovado / Approved: 15/07/2020 


\begin{abstract}
Being typically Brazilian and especially useful in the reprocessing of mozzarella, the Requeijão has become a product of high consumption and production in Brazil. Over time, the increased competitiveness among the dairy industries has improved the productivity of Requeijão. However, it is essential to increase the production and productivity of Requeijão without losing quality. It is known that a way to promote high productivity and high quality in a process is by using the Statistical Quality Control (SQC), which aims to help the production process to correct the identifiable errors throughout the process, in order to increase the profitability of production. This paper presents the process of Statistical Process Control (SPC) implementation in a Requeijão production line of a dairy industry in southern Minas Gerais. Control charts were used for variables and capability indexes, to monitor critical variables of the Requeijão production process, in order to meet the specifications of the Technical Regulation on Identity and Quality. After analysis of the results, it was found that the process variables are under statistical control and are capable to meet the specified standards. However, by analyzing the variability of the data, non-randomness patterns were identified, which indicates that the process variability is not under statistical control. The main cause which was pointed out was the poor accuracy of the data collected in the production process, which shows the effectiveness of SPC tools.
\end{abstract}

Keywords: quality management; milk derivatives; capability index.

\section{INTRODUÇÃO}

O avanço no consumo de requeijão no Brasil ocorre devido às suas características culinárias e por ser um produto muito requisitado, desde o café da manhã até no prato principal do jantar, o que o torna altamente procurado para ser processado em indústrias de laticínios (PAIVA et al., 2018).

$O$ requeijão é definido como sendo um tipo de queijo fundido, com duas variantes: o requeijão cremoso e o de manteiga (VIEIRA et al., 2014). O requeijão cremoso é obtido pela fusão de uma massa coalhada por coagulação ácida e adição de creme. Outros derivados lácteos como manteiga e/ou gordura anidra de leite (ou butter oil) podem ou não ser adicionados, desde que não contenham gordura ou proteína de origem não láctea. Para a sua comercialização o requeijão deve possuir requisitos físico-químicos característicos como gordura no extrato seco mínima de $55 \%$ e umidade máxima de $65 \%$ (BRASIL, 1997).

Os laticínios têm levado renda e emprego nos meios rural e urbano com mais de 1.187 instalações com registro no Sistema de Inspeção Federal (SIF). A atuação destes laticínios no comércio pode ser distribuída da seguinte forma: $75 \%$ em nível nacional, 10,3\% no próprio estado, $8,8 \%$ em outros estados e $5,9 \%$ para exportação. Além disso, a maior parte dos laticínios está localizada na região sudeste (638 laticínios) e a menor parte está no norte do país, com 91 estabelecimentos, sendo o queijo, a manteiga e o requeijão os produtos mais produzidos entre eles (LIMA et al., 2017).

Com o aumento de indústrias de laticínios no Brasil, sendo a maior parte de micro e pequeno porte, existe uma preocupação com a falta de ações estratégicas para que estes laticínios se tornem mais robustos perante o mercado e se destaquem na 
competitividade (LIMA et al., 2017). Com isso surge a necessidade de os laticínios buscarem por ferramentas que possibilitem obter produtividade sem renunciar à qualidade. Uma ferramenta que vem cumprir tal objetivo é o Controle Estatístico da Qualidade (CEQ) (FERREIRA; OLIVEIRA, 2008).

Uma das áreas de destaque no CEQ é o Controle Estatístico de Processo (CEP), que é a parte mais quantitativa do CEQ, sendo definido como um sistema de inspeção por amostragem que possui um conjunto de ferramentas de acompanhamento da qualidade, onde estas ferramentas possibilitam obter uma descrição detalhada do comportamento do processo, identificando sua variabilidade e possibilitando seu controle ao longo do tempo (LOPES et al., 2018).

Trabalho de Fontanini et al. (2003) no envase do leite em um laticínio em Santa Catarina utilizou cartas de controle da média e desvio padrão, junto com uma análise estatística multivariada, para diminuir a quantidade de variáveis do processo. Essa abordagem possibilitou mostrar que o processo se encontrava fora de controle estatístico e necessitava de um acompanhamento sistemático de manutenção da máquina de envase.

Rodrigues et al. (2019), realizaram um estudo de caso em um laticínio no processo de envase do leite. Neste caso foi aplicado a folha de verificação, fluxograma, histograma e as cartas de controle, podendo assim certificar que o processo de envase estava sob controle estatístico.

Alecrim (2015), em seu trabalho de conclusão de curso, fez o estudo da aplicação de CEP no envase de iogurte de morango em um laticínio na Paraíba, com o objetivo de identificar as causas de variabilidade do processo analisado de mês em mês. Seus resultados mostraram pontos fora de controle e de especificação que foram estudados através das cartas de controle e diagrama causa e efeito.
Lopes et al. (2018) realizaram o monitoramento do processamento de leite longa vida por meio do CEP em um laticínio em Ji-Paraná, RO, o que trouxe benefício à fabricação do produto.

Ribeiro et al. (2018) aplicaram cartas de controle paramétricas e não paramétricas em um estudo no recebimento do leite de uma queijaria de São Luís de Montes Belos, GO.

Com potencial de aplicação na produção de requeijão, uma das principais ferramentas do CEP, as cartas de controle, têm como objetivo monitorar os processos por meio de medições das variáveis de interesse, registrando os dados coletados ao longo do tempo em um gráfico de controle (FONTANINI et al., 2003).

Quando os pontos do gráfico estiverem dentro dos limites de controle e a disposição destes for aleatória, o processo é considerado sob controle estatístico (WERKEMA, 1995). Porém, se os pontos saírem dos limites de controle, ou não demonstrarem uma distribuição aleatória, o processo é julgado estando fora de controle. Uma ferramenta que possibilita detectar pontos fora de controle são os padrões de não aleatoriedade (NOMELINI et al., 2009).

Os padrões de não aleatoriedade são ferramentas de análise e interpretação dos pontos plotados nas cartas de controle para a verificação da estabilidade do processo. Os pioneiros a propor estes padrões foram: Shewhart em 1931, Western Electric em 1958 e Nelson em 1985. O objetivo é melhorar a eficiência em descobrir padrões de não aleatoriedade provenientes de causas especiais na linha de produção (NOMELINI et al., 2009).

Em um processo produtivo de requeijão surge a necessidade de avaliar se o processo é capaz de atender às especificações estabelecidas por órgãos reguladores. Esta avaliação é denominada de Capacidade do Processo, que consiste em medir a variabilidade natural 
do processo em relação a variabilidade permitida a esse processo, obtida nos limites de especificação (GONÇALVES; WERNER, 2009). No segmento de laticínios, o principal órgão inspecionador é o Sistema de Inspeção Federal (SIF), que é o responsável por assegurar a qualidade de produtos de origem animal comestíveis ao consumidor final.

Identificar o fator que ocasionou as causas especiais no processo é uma difícil tarefa que pode ser solucionada pelo uso do diagrama de Ishikawa ou Espinha de Peixe, que é um recurso gráfico usado para monitorar e mostrar as relações ligadas entre causas primárias e secundárias, onde uma está diretamente relacionada com a outra, uma relação de causa e efeito (FERREIRA; OLIVEIRA, 2008).

Desta forma, neste trabalho objetivouse aplicar as cartas de controle a variáveis críticas do processo de produção de requeijão de um laticínio situado no sul de Minas Gerais, com o intuito de verificar se estão sob controle estatístico e qual é sua capacidade de atender as especificações da legislação pertinente.

\section{MATERIAL E MÉTODOS}

O laticínio parceiro do trabalho localizado no Sul de Minas Gerais possui uma capacidade média de processamento de 1.000 litros de leite por dia. Entre os produtos que o laticínio produz, foi escolhido o requeijão cremoso para ser analisado, e sua produção possui 4 formatos: copo de $200 \mathrm{~g}$, bisnagas de $1,8 \mathrm{~kg}$ e $400 \mathrm{~g}$, e balde de $3,6 \mathrm{~kg}$.

No início foi realizado um estudo junto ao laticínio para se identificar quais são as possíveis variáveis críticas na produção de requeijão e quais o laticínio já realizava as suas medições. Foram elencadas quatro variáveis: $\mathrm{pH}$ da massa, teor de gordura no extrato seco $(\%)$, umidade $(\%)$ e tempo de processo (h).
Em pesquisa junto a equipe de produção foi identificado que o requeijão bisnaga $400 \mathrm{~g}$ é o mais fabricado e, portanto, foram utilizados os dados da produção deste produto para monitorar as variáveis do processo por meio das cartas de controle.

A coleta dos dados das variáveis críticas do requeijão bisnaga $400 \mathrm{~g}$ ocorreu em um período de sete meses, sendo de janeiro a julho de 2019, com uma frequência de produção média de quatro vezes ao mês. Após análise do requeijão bisnaga $400 \mathrm{~g}$, todo o processo foi repetido para os produtos requeijão de copo $200 \mathrm{~g}$ e bisnaga $1,8 \mathrm{~kg}$, porém, para simplificar os resultados, apresentaremos somente o estudo realizado no requeijão bisnaga $400 \mathrm{~g}$.

Para a obtenção das cartas de controle e da capacidade do processo foi utilizado o software R com o uso do pacote CEPpt desenvolvido por Bastos e Ferreira (2012). Para montar o diagrama causa e efeito foi utilizado o software R com o pacote Quality Control Charts (QCC), versão 2.7, com a função cause.and.effect (R CORE TEAM, 2019).

\section{Controle Estatístico de Processo}

\section{Cartas de controle}

As cartas de controle utilizadas neste trabalho foram a medida individual e a amplitude móvel, pelo fato de as variáveis críticas do processo terem apenas uma observação por produção. Esta carta tem sua média centrada em todos os $m$ subgrupos racionais e possui limite superior de controle (LSC) e limite inferior de controle (LIC), estabelecidos a três desvios-padrão do valor esperado para a variável monitorada (FERREIRA; OLIVEIRA, 2008).

Para a carta amplitude móvel $(A M)$ com linha central $L C_{A M}$, o parâmetro de dispersão utilizado é a amplitude móvel média $(A M)$, definida pela Equação 1 . 


$$
\begin{aligned}
L C_{A M}=\underline{A M}=\frac{\sum_{j=1}^{m-1} A M_{j}}{m-1} \\
L S C_{A M}=3,268 \cdot \underline{A M}
\end{aligned}
$$

(Equação 1)

onde AM é definido como amplitude móvel $A M_{i}=\left|x_{i}-x_{i-1}\right|, \mathrm{i}=2,3, \ldots, \mathrm{m} ;$ e m é o número de amostras. $L C_{A M}$ é a linha central, $L S C_{A M}$ é o limite superior de controle e $L I C_{A M}$ é igual a zero para a carta de controle amplitude móvel.

Para a carta de medida individual (X) temos que a linha central $L C_{X}$ e os limites de controle $L S C_{X}$ e $L I C_{X}$ são designados pela Equação 2.

$L S C_{X}=\underline{X}+2,66 \underline{A M}$

$L C_{X}=\underline{X}$

$L I C_{X}=\underline{X}-2,66 \underline{A M}$

(Equação 2)

onde $\underline{X}$ é a média dos dados e $\underline{A M}$ é a média das amplitudes móveis (FERREIRA; OLIVEIRA, 2008).

\section{Padrões de não aleatoriedade (PNA)}

Segundo Nomelini et al. (2009), os testes que podem ser analisados nos padrões de não aleatoriedade são os testes de Shewhart (1931), Western Electric (1958) e Nelson (1985) que são definidos como oito critérios, descritos abaixo:

Critério 1: Pontos acima do LSC ou abaixo do LIC.

Critério 2: Nove pontos, em sequência, abaixo ou acima da LC.

Critério 3: Seis pontos em sequência crescente ou decrescente.

Critério 4: Quatorze pontos consecutivos alternando-se no gráfico.

Critério 5: Dois em três pontos consecutivos na zona A.

Critério 6: Quatro em cinco pontos consecutivos na zona A ou B.

Critério 7: Quinze pontos consecutivos na zona C.

Critério 8: Oito pontos consecutivos de ambos os lados da LC, nenhum deles na zona C.

Para encontrar as causas especiais do processo, os padrões de não aleatoriedade se baseiam nas seis zonas que existem entre os limites de controle, com a dimensão de 1 desvio padrão, chamados zonas $\mathrm{A}, \mathrm{B}, \mathrm{C}, \mathrm{C}$, $\mathrm{B}$ e A que estão distribuídas simetricamente com a média LC, como mostra a Figura 1 (NOMELINI et al., 2009).

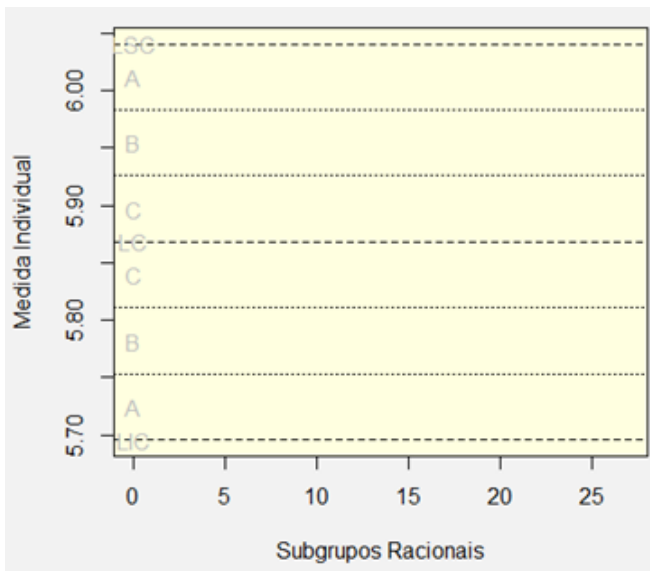

Figura 1 - Modelo de carta de controle, com as respectivas zonas para os testes de não aleatoriedade

Todos os oito critérios são utilizados em conjunto na hora da busca por padrões de não aleatoriedade nas cartas de controle. Quando um destes critérios é atendido entende-se que existe uma causa especial de variação no processo não normal.

\section{Capacidade do Processo}

\section{Índice Cpk}

Para a capacidade do processo foi utilizado o índice de desempenho $C p_{k}$ que, segundo Gonçalves e Werner (2009), leva em consideração a distância média do processo em relação aos limites de especificação, e é definido conforme Equação 3. 
$C p_{k}=\min \left(\frac{L S E-\mu}{3 \sigma}, \frac{\mu-L I}{3 \sigma}\right)$

(Equação 3)

onde $\mu$ e $\sigma$ é a média do processo e o desviopadrão respectivamente.

O índice $C p_{k}$ associa a variabilidade permitida ao processo com a variabilidade natural do processo, e com isso se torna possível definir que, quanto maior for o valor de $C p_{k}$, maior será a capacidade do processo em satisfazer as especificações. A análise do índice $C p_{k}$ pode ser feita através das relações abaixo onde Gonçalves e Werner (2009) especificam os intervalos de referência para o índice.

$$
\begin{gathered}
C p_{k}<1 \text { Processo Incapaz } \\
1 \leq C p_{k} \leq 1,33 \text { Processo Capaz } \\
C p_{k} \geq 1,33 \text { Processo Potencialmente Capaz }
\end{gathered}
$$

\section{Diagrama Causa e Efeito}

Com o intuito de encontrar as possíveis causas especiais de variação foi utilizado o diagrama de Causa e Efeito, de Ishikawa ou Espinha de Peixe. Ele atuou como um guia para a identificação das causas que geraram os problemas observados e assim facilitar a sua correção. A sua construção pode ser realizada por meio das seguintes etapas (FERREIRA; OLIVEIRA, 2008):

Etapa 1: Definir o problema a ser analisado; Etapa 2: Reunir uma equipe para estudar as causas do problema;

Etapa 3: Montar a linha central do diagrama; Etapa 4: Especificar as causas potenciais e ligá-las a linha central;

Etapa 5: Classificar as possíveis causas em tópicos;

Etapa 6: Ordena as causas listadas pela probabilidade de ocorrência;

Etapa 7: Adotar ações corretivas.

Após ter essas informações listadas devese distribuí-las dentro do diagrama espinha de peixe (FERREIRA; OLIVEIRA, 2008).
O diagrama foi utilizado neste trabalho para elencar as possíveis causas de variação da gordura e do $\mathrm{pH}$ da massa, como forma de facilitar a visualização das possíveis causas de variação. Estas causas foram levantadas em reunião conjunta com a equipe de produção, por meio da análise inicial dos resultados das cartas de controle.

\section{RESULTADOS E DISCUSSÃO}

\section{Cartas de Controle}

Analisando as variáveis críticas do processo: $\mathrm{pH}$, gordura (\%), umidade (\%) e tempo de processo, presentes nas Figuras $2 a$, $2 c, 2 e$ e $2 g$, pode-se observar que não houve presença de padrões de não aleatoriedade e nenhum critério a ser destacado como causa especial, o que demonstra que as variáveis se encontram sob controle estatístico.

Nas cartas amplitude móvel foi possível destacar alguns critérios dos padrões de não aleatoriedade. Na variável pH (Figura 2b) foram encontrados nove pontos em sequência abaixo da linha central (LC), critério 2 dos PNA, devido a um padrão gráfico (M) desenhado nesta região. Para a variável umidade (Figura 2f), existe um (W) desenhado por 6 pontos abaixo da LC, se enquadrando no critério 3 dos PNA. Na variável tempo de processo destaca-se 12 pontos decrescendo abaixo da LC, como mostra a Figura $2 h$, o que não era de se esperar para uma distribuição que deveria se comportar de forma aleatória.

O que é comum para a carta de controle amplitude móvel destas variáveis, por apresentar semelhança na variabilidade dos dados, é uma baixa precisão na hora da coleta das medidas, o que remete que a variabilidade dos dados não se encontra sob controle estatístico e pode estar mascarando o controle estatístico das medidas individuais das variáveis.

Na carta de amplitude móvel da variável 
gordura, é possível destacar cinco pontos em cima do limite inferior de controle (LIC), critério 1 dos PNA, como mostra a Figura $2 d$. Foram detectados pontos de variabilidade zero, o que significa que 5 medições de gordura tiveram o mesmo valor, o que está representado pelas setas na (Figura 2c), confirmando a hipótese de baixa precisão nas medidas.

Em análise aos resultados das cartas de controle, a principal hipótese de pontos com variabilidade zero e padrões gráficos são corroboradas pelo trabalho de Chioderoli et al. (2011), que apresentou vários pontos de variabilidade zero nas cartas amplitude móvel e alguns pontos fora dos limites de controle durante a medição da produção de grãos de soja, o que o autor coloca estar relacionado principalmente à regulagem incorreta da colhedora e a falta de experiência do operador, no manuseio do equipamento.

De forma semelhante, o trabalho de Takahashi et al. (2012) destaca vários pontos zero na carta amplitude móvel obtida na medição da qualidade do leite em várias fazendas em épocas distintas do ano, sendo consequência, principalmente, da baixa qualidade com o trato dos animais.

Dantas (2015), em seu trabalho de aplicação do CEP no envase do leite pasteurizado em um laticínios do Rio Grande do Norte, verificou padrões gráficos nas cartas de controle aplicado ao envase e pontos de variabilidade zero, onde este destaca serem as principais causas: desregulagem nos instrumentos de medidas e a constante atuação do operador na tentativa de ajustar o processo. Com esses destaques dos trabalhos citados, a hipótese principal do presente trabalho é mantida junto com a proposta de Dantas (2015), que aponta a constante atuação do operador em corrigir o processo como possível causa dos PNA na amplitude dos dados deste trabalho.

Prezar por uma melhor qualidade na coleta das informações e na rotina do operador são causas primordiais que precisam ser analisadas no processo produtivo de requeijão pela gerência, para não obter a falsa impressão de que os dados das variáveis críticas do processo estão fora de controle estatístico.

De cunho prático, com o repasse das informações para o laticínio, foi obtida a informação de que a variável $\mathrm{pH}$ do requeijão era corrigida pelo operador durante o processo até se obter o valor esperado. Além disso, a falta de precisão nas medidas não gera transtornos na produção, devido à baixa produção ocasional sob demanda.

Como forma de solução à falta de variabilidade dos dados, foi proposto que a precisão dos instrumentos de medida fosse revisada e que o monitoramento do processo produtivo de requeijão fosse analisado para confirmar a hipótese de que ajustes contínuos durante o processo de produção estão limitando a variação das medidas dos dados, o que é muito claro de se observar no gráfico $c$ da Figura 2, onde parece que a variável gordura está limitada ao valor de $60 \%$. Portanto, repetir a medida mais de uma vez durante o dia de produção, ajustar a precisão dos instrumentos de medida, assim como instruir e treinar os funcionários do processo produtivo, pode solucionar a não aleatoriedade encontrada no gráfico da amplitude móvel e colocar o processo novamente sob controle estatístico.

\section{Capacidade do Processo}

O principal objetivo da aplicação do índice de capacidade foi medir se o requeijão atende as normas do seu Regulamento Técnico de Identidade e Qualidade (RTIQ), que regulamenta o processo produtivo de requeijão bisnaga 400 g a obter: gordura no extrato seco maior ou igual a $55 \%$, e umidade menor ou igual a $65 \%$ (BRASIL, 1997). Além disso, foi monitorado o $\mathrm{pH}$, entre os limites de 5,65 a 5,95 . 


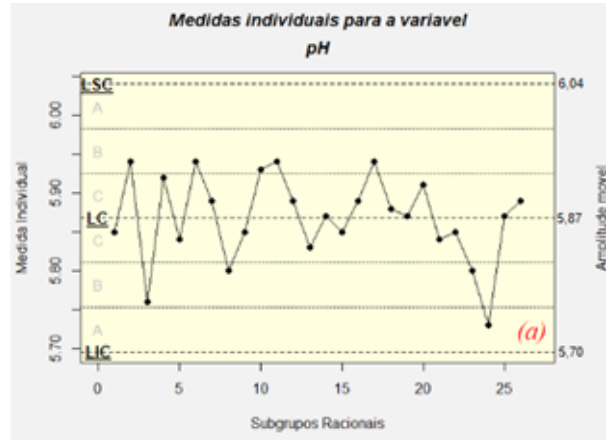

Medidas individuais para a variavel gordura (\%)

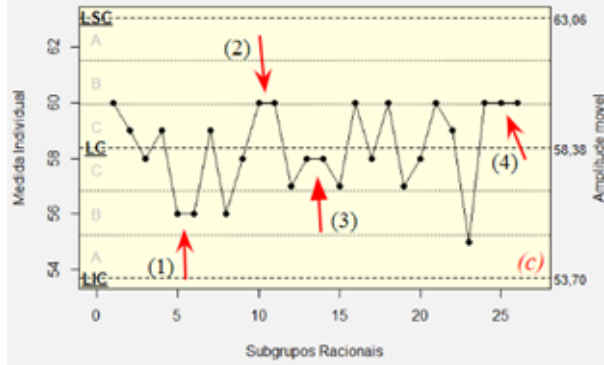

Medidas individuais para a variavel umidade $(\%)$

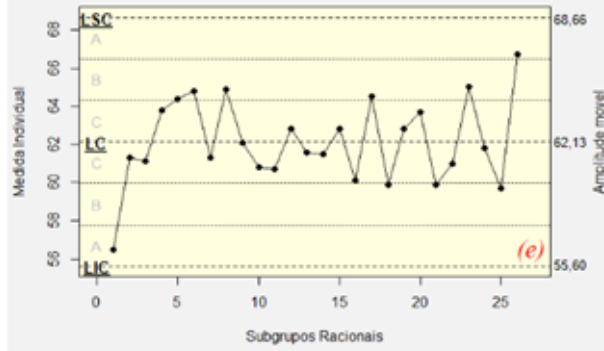

Medidas individuais para a variavel tempo (h)

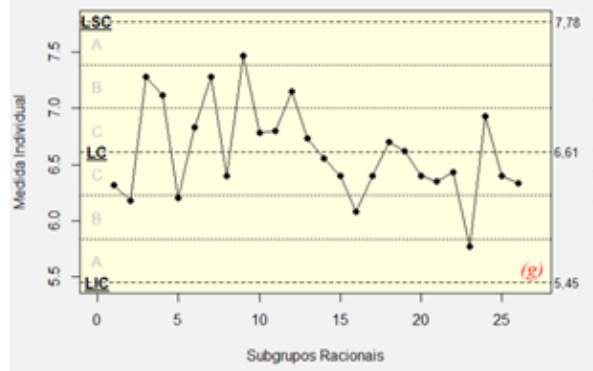

Amplitude Movel para a variavel

$\mathrm{pH}$

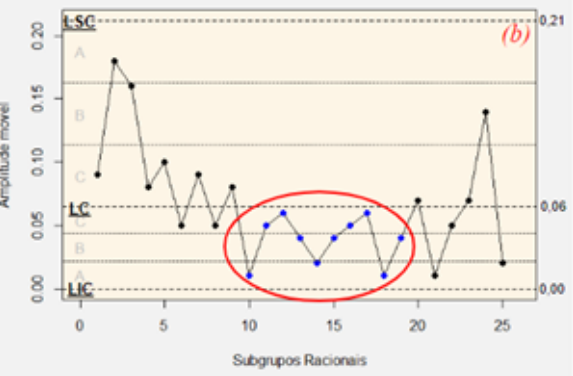

Amplitude Movel para a variavel gordura $(\%)$

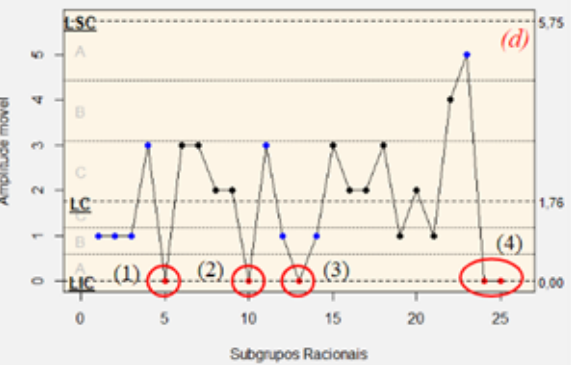

Amplitude Movel para a variavel umidade $(\%)$

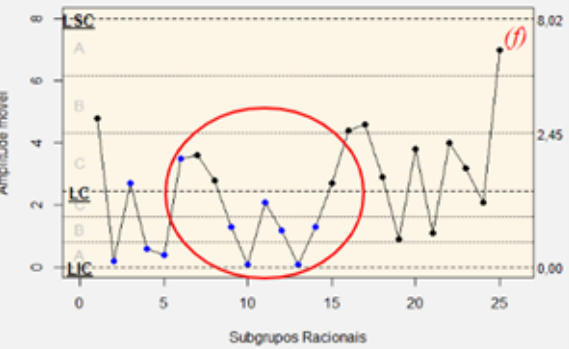

Amplitude Movel para a variavel

tempo (b)

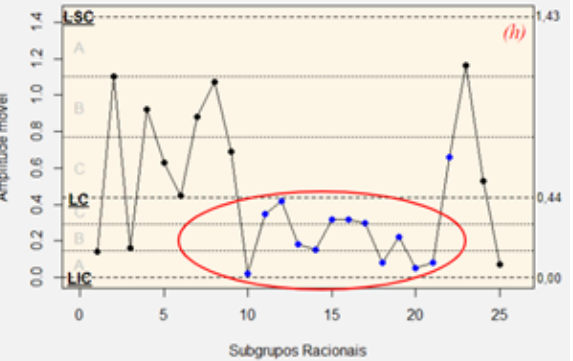

Figura 2 - Cartas de controle medida individual e amplitude móvel, para as variáveis: $\mathrm{pH}$ ( $a$ e $b$ ), gordura ( $c$ e $d$ ), umidade ( $e$ e $f)$ tempo de processo $(g$ e $h)$ respectivamente, do requeijão bisnaga $400 \mathrm{~g}$ 

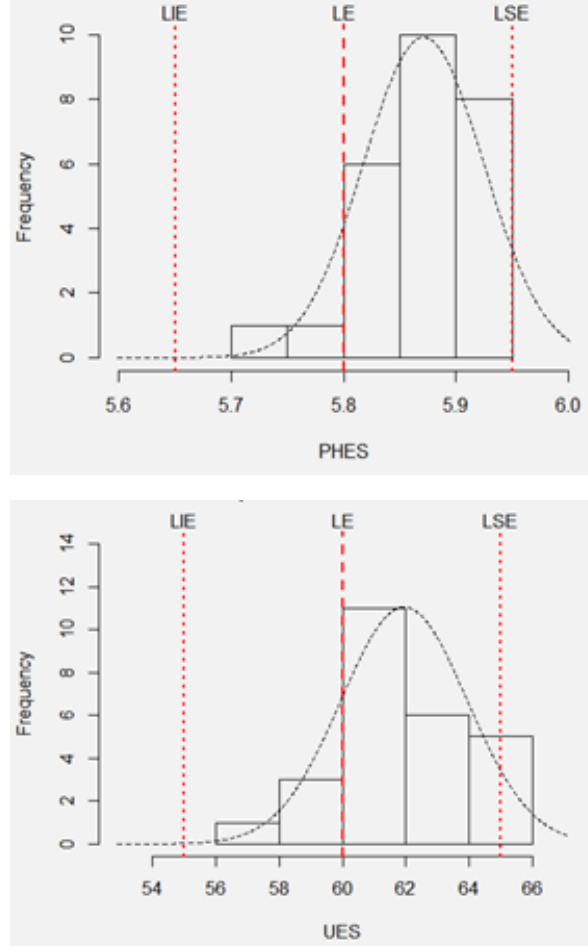
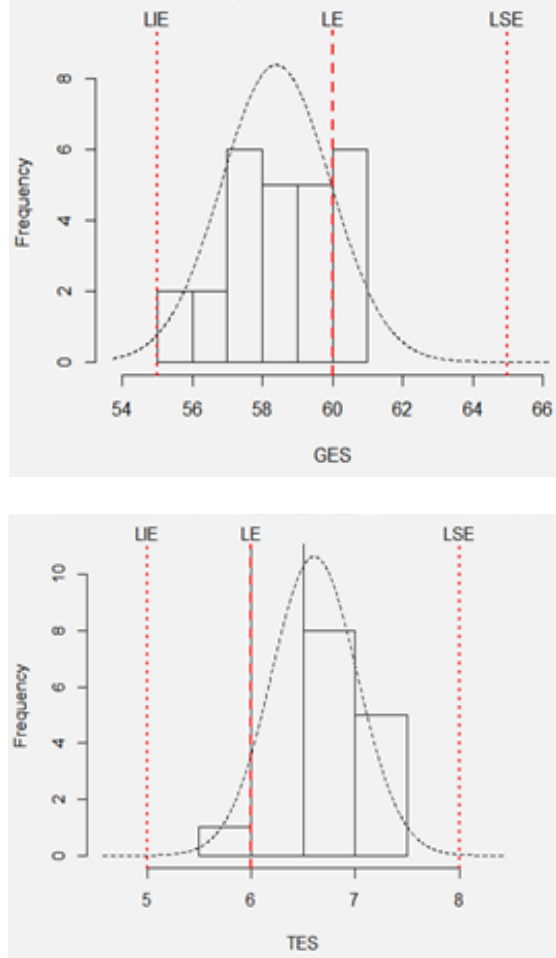

Figura 3 - Capacidade de processo das variáveis: pH (PHES), gordura no extrato seco (GES), umidade (UES) e tempo de processo (TES)

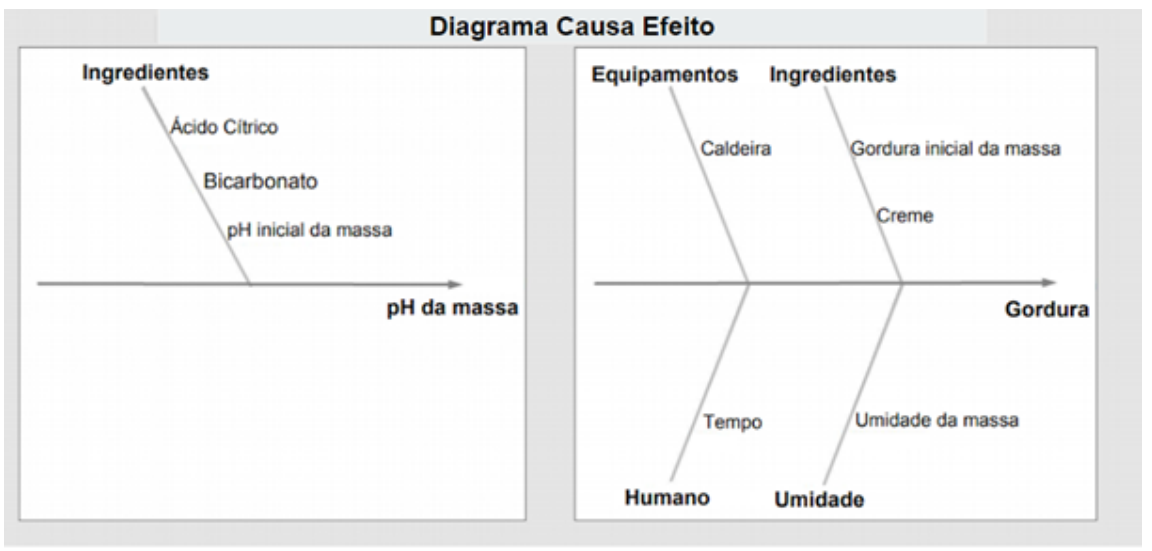

Figura 4 - Diagrama causa efeito das duas principais variáveis inerentes ao processo produtivo de requeijão 
Para os gráficos da Figura 3 foram utilizados o índice para análise da capacidade do processo. As variáveis $\mathrm{pH}$ da massa (PHES), gordura no extrato seco (GES) e umidade (UES) obtiveram índice de 1,14; 39,65 e 39,64, respectivamente, mostrando ser capaz em atender ao seu RTIQ. A variável tempo de processo (TES) se encontra capaz de obedecer às médias e os limites de controle do processo, obtendo índice de 18,82.

Em destaque para os gráficos GES e UES da Figura 3, é possível notar uma correlação negativa entre as variáveis gordura no extrato seco e umidade. Tal fato foi verificado com a equipe de produção e constatada a correlação negativa como natural do processo, visto que um maior teor de umidade preconiza um menor teor de sólidos, do qual a gordura faz parte.

Vale ressaltar que processos que atendem as normas de especificação não necessariamente se encontram sob controle estatístico. É preciso ter o devido cuidado para não deixar que os limites de especificação diminuam o rigor e a confiabilidade dos dados, simplesmente por ter que atender normas. O processo deve prezar pela busca da qualidade e dessa forma atender as normas se tornará algo trivial (VIEIRA et al., 2014).

\section{Diagrama Causa e Efeito}

Com o intuito de verificar quais são as possíveis causas especiais que estão atuando sobre o processo, foi desenvolvido um diagrama causa e efeito para as variáveis: $\mathrm{pH}$ da massa e Gordura/Umidade, baseado nas informações obtidas dos colaboradores do laticínio, como mostra a Figura 4.

$\mathrm{O}$ pH da massa depende basicamente da quantidade de produtos adicionados à receita (ácido cítrico, bicarbonato e o pH inicial da massa), sendo a sua variação controlada por estes ingredientes mais um erro humano que possa variar estas quantidades. Em conversa com a produção, nos foi passado que o $\mathrm{pH}$ da massa no começo do cozimento é o fator primordial, que irá definir se vai ser acrescentado ingredientes para correção do pH no final da massa ou não.

A variação percentual da gordura depende de quatro fatores: Humano, Ingredientes, Equipamentos e Umidade. No fator humano, pode-se observar que quanto maior o tempo de cozimento da massa, menor é o valor da umidade e maior será a gordura final. Este fator entrou no diagrama devido a produção sob demanda ocorrer algumas vezes na semana, podendo o requeijão ficar maior tempo exposto ao cozimento e assim variar a sua gordura final de forma significativa. A caldeira é um fator significativo no cozimento do requeijão, de forma que se houver variação no fornecimento de vapor, pode atrasar o cozimento e consequentemente variar o valor final da gordura.

\section{CONCLUSÃO}

O uso das cartas de controle, capacidade do processo e o diagrama causa e efeito se mostrou um conjunto de ferramentas eficiente para mostrar que os processos se encontram sob causas especiais de variação, possibilitando interligá-las com o cotidiano da produção. Por fim, as variáveis críticas do processo foram analisadas de forma individual e o $\mathrm{pH}$ da massa, gordura no extrato seco e umidade se apresentaram dentro dos limites estabelecidos.

Os padrões de não aleatoriedade denunciaram uma baixa precisão nos dados, o que pode estar mascarando o controle estatístico das medidas individuais das variáveis. Logo, uma melhora na precisão dos dados e das medidas junto com o uso do diagrama causa e efeito e o treinamento da produção com relação ao processo é uma solução cabível de ser realizada para eliminar as causas especiais presentes no processo. 
Para trabalhos futuros, indicamos realizar uma nova busca por variáveis críticas do processo produtivo de requeijão com o intuito de explicar o rendimento do processo, o que pode auxiliar na pesquisa e melhora do processo produtivo de requeijão.

Conclui-se que a partir da aplicação do CEP ao processo produtivo de requeijão pode ser averiguado que a variabilidade do processo mostrou-se fora de controle, porém as variáveis foram capazes de atender as especificações do RTIQ, mostrando a efetividade do CEP aplicado a produção de requeijão.

\section{REFERÊNCIAS}

ALECRIM, D. A. Aplicação do controle estatístico do processo no envase de iogurte em uma indústria de laticínios na Paraíba. 2015. 72 f. (Trabalho de Conclusão de Curso Engenharia de Produção) - Universidade Federal de Campina Grande, Sumé, 2015.

BASTOS, R. L.; FERREIRA, E. B. CEPpt: Um pacote $\mathrm{R}$ para o Controle Estatístico de Processo. Revista Sigmae, v. 1, n. 1, p. 1-17, 2012.

BRASIL. Ministério da Agricultura e do Abastecimento. Portaria $n^{\circ} 359$, de 4 de setembro de 1997. Regulamento Técnico para Fixação de Identidade e Qualidade do Requeijão ou Requesõn. Diário Oficial da República Federativa do Brasil: seção 1, Brasília, DF, n. 172, p. 19.690, 08 set. 1997.

CHIOdEROLi, C. A. et al. Perdas de grãos e distribuição de palha na colheita mecanizada de soja. Revista Bragantia, v. 71, n. 1, p. 112-121, 2012. DOI: 10.1590/ S0006-87052012005000003.

\section{DANTAS, M. B. Aplicação do CEP no envase} do leite pasteurizado de uma indústria de laticínios do RN. 2015. 30 f. (Trabalho de Conclusão de Curso Tecnólogo em Alimentos) - Instituto Federal de Educação, Ciência e Tecnologia do Rio Grande do Norte, Currais Novos, 2015.

FERREIRA, E. B.; OLIVEIRA, M. S. Controle Estatístico da Qualidade. Lavras: UFLA/FAEPE, 2008. 87 p.

FONTANINI, C. A. C. et al. O uso da Análise de Componentes Principais e dos gráficos de Controle Estatístico de Processos numa indústria de laticínios do Planalto Serrano de Santa Catarina. Revista EnANPAD, v. 1, n. 1, p 1-11, 2003.

GONÇALVES, P. U.; WERNER, L. Comparação dos índices de capacidade do processo para distribuições nãonormais. Gestão \& Produção, v. 16, n.1, p. 121-132, 2009. DOI: 10.1590/S0104$530 \times 2009000100012$

LIMA, L. P.; PEREZ, R.; CHAVES, J. B. P. A indústria de laticínios no Brasil - Um estudo exploratório. Boletim Centro de Pesquisa de Processamento de Alimentos, v. 35, n. 1, p. 1-13, 2017. DOI: 10.5380/cep.v35i1.55942

LOPES, G. G. et al. Monitoramento dos pontos críticos de controle aplicado no processamento do leite longa vida em indústria de laticínios de Ji-Paraná. Revista Veterinária Notícias, v. 24, n. 2, p. 1-14, 2018.

NELSON, L. S. Interpreting shewhart X-bar control charts. Journal of Quality Technology, v. 17, n. 2, p. 114-118, 1985. DOI: $10.1080 / 00224065.1985 .11978945$

NOMELINI, Q. S. S.; FERREIRA, E. B.; OLIVEIRA, M. S. Estudos dos padrões de não aleatoriedade dos gráficos de controle de 
Shewart: um enfoque probabilístico. Gestão \& Produção, v. 16, n.3, p. 414-421, jul.-set. 2009. DOI: $10.1590 / \mathrm{S} 0104-530 \mathrm{X} 2009000300008$

PAIVA, A. R et al. Efeito da temperatura na viscosidade aparente de diferentes marcas de requeijão cremoso tradicional e light. Revista do Instituto de Laticínios Cândido Tostes, v. 73, n. 4, p. 209-219, 2018. DOI: $10.14295 / 2238-6416 . v 73 i 4.689$

R DEVELOPMENT CORE TEAM. R: a language and environment for statistical computing. Vienna: R Foundation for Statistical Computing, 2019. Disponível em: http://www.rproject.org

RIBEIRO, L. H. M. S. et al. Controle estatístico de processo paramétrico e não paramétrico em uma indústria de laticínios. Revista do Instituto de Laticínios Cândido Tostes, v. 73, n. 3, p. 112-121, 2018. DOI: 10.14295/2238-6416.v73i3.696

RODRIGUES, L. C. et al. Controle estatístico da qualidade: Um estudo de caso em um Laticínio. In: ENCONTRODE ENGENHARIA DE PRODUÇÃO AGROINDUSTRIAL, 13., 2019, Campo Mourão. Anais [...]. Campo Mourão: Universidade Estadual de Maringá, 2019. Disponível em: http://www.dep.uem.br/ gdct/index.php/simeprod/article/view/1652. Acesso em: dez. 2019.

SHEWHART, W. A. Economic Control of Quality of Manufactured Product. New York: D. Van Nostrand, 1931.

TAKAHASHI, F. H. et al. Variação e monitoramento da qualidade do leite através do controle estatístico de processos. Revista Ciência Animal Brasileira, v. 13, n. 1, p. 99-107, 2012. DOI: $10.5216 / \mathrm{cab}$. v13i1.14870

VIEIRA, M. C. et al. Análise de viabilidade econômica da produção de formulações de requeijão cremoso sem adição de gordura e com teor reduzido de sódio. Revista Informações Econômicas, v. 44, n. 3, p. 37-50, 2014.

WERKEMA, M. C. C. Ferramentas da qualidade: Ferramentas estatísticas básicas para o gerenciamento de processos. Belo Horizonte: QFCO, 1995.

WESTERN ELETRIC COMPANY. Statistical Quality Control Handbook. 2nd. ed. Indianapolis: Western Electric Company, 1958. 